\title{
Finding Markers in Amyotrophic Lateral Sclerosis Diagnosis
}

\author{
Barbara Aymee Hernandez* \\ Centro de Neurociencias de Cuba, Havana, Cuba
}

${ }^{*}$ Corresponding author: Barbara Aymee Hernandez, Centro de Neurociencias de Cuba, Havana, Cuba, Tel: +53 $72087090,+57$ 2087091; Email: barbara@cbeuro.edu.cu

Rec date: Dec 21, 2017; Acc date: Jan 10, 2018; Pub date: Jan 12, 2018

Citation: Hernandez BA (2018) Finding Markers in Amyotrophic Lateral Sclerosis Diagnosis. J Neurol Neurosci Vol.9 No.1:239.

\section{Abstract}

Amyotrophic Lateral Sclerosis (ALS) is an uncommon illness, it is caused by moto neuron degeneration, upper, lower and bulbar muscles are affected. Some research also report degeneration in no motor structures of the brain. We proposed to evaluate Electrophysiological and Image techniques like markers in ALS diagnosis and correlate these results. During January 2015 to January 2017, twenty patients with ALS diagnosis and twenty health subjects were evaluated. Sensory and by motor nerve conduction studies, Electromyography, SomatoSensory Evoked Potentials were done to the patients. 3T MRI image were obtained from the patients and from the health subjects. Post processing MRI techniques like voxel based morphometric, diffusion techniques and corticospinal tract and corpus callosum tractography were applied at different levels of the brain structures. Nerve conduction study was positive in $90 \%$ of the patients, SSEP were positive in $60 \%$ and EMG abnormalities were observed in $100 \%$ of patients. Anatomic MRI was positive in $50 \%$ of the patients. Fractional Anisotropy was reduced in ALS group in comparison with health group, more significant at cortex, internal capsule and corpus callosum. Fibers number of cortico-spinal tract and corpus callosum were diminished in ALS group in relation to health group. Also grey and white matter were reduce in ALS group, in areas such as: cingulate gyrus, anterior portion of occipital lobe, left caudate and putamen nucleus, right claustrum nucleus, lower and medium temporal gyrus bilateral, left precentral and post-central gyrus, corpus callosum, corticospinal tract, bilateral internal capsule, bilateral optical radiation, bilateral lower longitudinal fascicle, bilateral hippocampal fimbriae, bilateral radiated corona and pontocerebellar fibers. Electrophysiological studies confirmed ALS diagnosis in $100 \%$ of cases. MRI methods show abnormalities in motor and not motor structures of brain in ALS patients. They could be markers in early ALS diagnostic.

Keywords: Corticospinal tract; Postcentral gyrus; Corpus callosum; Electromyography; Amyotrophic lateral sclerosis

\section{Introduction}

Amyotrophic Lateral Sclerosis (ALS) is a fatal and rapidly progressing neurodegenerative disease affecting both upper and lower motor neuron systems. It is characterized by involvement of both motor neurons, median survival time of 2-4 years from onset of symptoms. It is the third more frequent neurodegenerative diseases [1].

In Europe and the United States, ALS affects about 2 people per 100,000 habitants per year. In Cuba rates of ALS are unknown. Cuban National Neurologic and Neurosurgery Institute is ALS reference centre and it reports about 200 new ALS cases by year [2] recently have changed the widely held belief that ALS affects only the motor neuron system, because evidence suggests that ALS is a multisystem neurodegenerative disease that involves sensory and extrapyramidal systems [1-3].

Some researchers have been working looking forward markers for early diagnosis of the disease [4]. For example: Feneberg et al. have showed increase of TDP-43 (it is a phosphorylated and ubiquinated cytoplasmic aggregated of neurons and glial cells) in bio fluids and tissues in early step of ALS [5]. Audiutori et al. have isolated neurofilaments containing hetero-aggregates in blood of ALS patients [6]. Thompson et al. have determinate extracellular vesicles in Cerebrospinal Fluid (CSF) in ALS patients [7] for his part Blasso et al. have determinate circulating exosomes in biological fluids [8]. Aricha et al. have showed highs levels of chitotriosidase and Lipocalin 2 in CSF of ALS patients [9] other researches like Brenmer et al. have demonstrated abnormalities in the composition of faecal micro biome in ALS patients [10].

Neurophysiological studies are very useful in ALS diagnosis confirmation, but themselves they don't should be considered as a markers in ALS diagnosis. MRI has been used to reject other diagnosis possibilities that could mimic ALS. Nevertheless, new image processing as voxel based morphometric, cortical thickness, volumetric analysis, fractional anisotropy, tractography and others have showed interesting changes in brain structures of ALS patients [1,2].

With this research we purpose: Evaluate the utility of image studies in detection of ALS abnormalities and correlate image finding with clinic abnormalities in ALS patients. 


\section{Material and Methods}

\section{Subjects}

A total of 20 sporadic ALS patients were recruited between 2015 and 2017 for this study, they met the criteria for clinically definite or probable (El Escorial criteria). Twelve of them were male and eight were female. All of them were evaluated at Cuban Neuroscience Centre.

The age of the patients was from twenty five to seventy four years old, mean 53.3 years old.

The control group consisted of 20 sex- and age-matched healthy subjects. We obtained written informed consent form ALS and health control group to participate. The study protocol was approved by Ethical Committee of Cuban Neuroscience Centre and all of patients and health subjects were agree with the evaluation and signed informed consent.

\section{Inclusion criteria for patient group}

Met the El-Escorial criteria for clinically definite or probable ALS (upper and lower motor neuron signs in two or three anatomic regions, without image evidence of another neurologic disease).

- Sporadic form of ALS

- Age between 20 and 80 years old

- Both sexes

- Any race

- No evidence of other neurologic disease

- No evidence of respiratory failure

- Evolution time lower one year

\section{Exclusion criteria for patient group}

- Upper or lower motor neuron signs in only one anatomic region

- Upper or lower motor neuron signs due to another neurologic disease

- Patients with contraindication for MRI or neurophysiologic evaluation

- Patients with respiratory failure

- Patients that deny participating in the investigation

\section{Inclusion criteria for health subject group}

- Health subjects

- No antecedent of disease

- Negative clinic exam

- Age similar to ALS group

- Both sexes

- Any race

\section{Exclusion criteria for health subject group}

- Patients with contraindication for MRI or neurophysiologic evaluation
- Patients that deny participating in the investigation

\section{Clinical measurements}

ALS Functional Rating Scale Reviewed (ALSFRS-R) was used to evaluate the functional status of ALS patients based on 12 items, functional disability scores range from 0 (maximum disability) to 48 (normal) points.

\section{Neurophysiological tests}

Motor and Sensory nerve conduction studies were done to ALS patients, in order to evaluate main nerves of the extremities.

Somato Sensory Evoked Potentials (SSEP) of tibial nerve was done to ALS patients.

Main extremities and bulbar muscle were evaluated through needle electromyography (EMG) study in patients with ALS.

\section{Image acquisition}

MRI was carried out using a 3 T Allegra system scanner (Siemens) equipped with a standard quadrature head coil. High-resolution three-dimensional whole-brain

T1-weighted MRI scans were acquired using a magnetization prepared rapid gradient echo sequence, were obtained as a volumetric three-dimensional spoiled fast gradient echo with the following parameters: $T R=250 \mathrm{~ms}, \mathrm{TE}=2.6 \mathrm{~ms}$, slice thickness $=1.0 \mathrm{~mm}$; flip angle $=9 \hat{\mathrm{E}}$; and field of view $=230 \times$ $230 \mathrm{~mm}, 1 \times 1 \times 1 \mathrm{~mm}^{3}$ voxel size. The volume consisted of 192 contiguous coronal sections covering the entire brain.

T2-weighted MRI scans were acquired using the following parameters: $\mathrm{TR}=3500 \mathrm{~ms}, \mathrm{TE}=354 \mathrm{~ms}$.

FLAIR MRI scans were acquired using the following parameters: $\mathrm{TR}=12.000 \mathrm{~ms}, \mathrm{TE}=140 \mathrm{~ms}$.

DTI scanning study protocol consisted of 80 volumes, slice thickness $2.0 \mathrm{~mm}$, representing 80 gradient directions, $\mathrm{TR}=9400 \mathrm{~ms}, \mathrm{TE}=80 \mathrm{~ms}$, slice thickness $=2.0 \mathrm{~mm}$; flip angle $=$ $90, b=1000 \mathrm{~s} / \mathrm{mm}^{2}$ and two scan with gradient $0(b=0)$, resolution was $1 \times 1 \times 1$.

\section{Image processing}

DTI processing: DTI images were processed using SPM 5 statistical parametric mapping software (Welcome Trust Centre for Neuroimaging, UCL, London, UK) in the analysis environment MATLAB (version R200 8b) within a Mat lab framework (The Math Works Inc., Natick MA, USA). Images were reoriented into oblique axial slices aligned parallel to the anterior-posterior commissural axis with the origin set to the anterior commissure, Eddy currents distortions were corrected, diffusion tensor was estimated, scalar maps (FA) were constructed, fibre tracking was done, tensor were visualized.

VBM analysis: VBM was a fully automated, whole-brain technique that enables measurement of regional brain 
volumes based on voxel-wise comparison of grey and white matter volumes using Statistical Parametric Mapping 5 software, running on Mat lab 2008b.

VBM was performed by using SPM5 (Welcome Department of Imaging Neuroscience, London, United Kingdom) and the DARTEL registration method. Briefly, the process was as follows: T1-weighted images were segmented by using VBM5.1 toolbox of SPM, the images were imported in DARTEL, rigidly aligned, and segmented into grey and white matter, the grey and white matter segments were coregistered simultaneously by using the fast diffeomorphic image registration algorithm and the flow fields were created, the flow fields were then applied to the rigidly aligned segments to warp them to the common DARTEL space and then were modulated by using the Jacobian determinants, the modulated images from DARTEL were normalized to the MNI template by using an affine transformation estimated from the DARTEL grey matter template and the a priori grey matter probability map without resampling, before the statistical computations, the images were smoothed with an 10-mm FWHM Gaussian filter.

Grey and white matter of ALS patients and health subjects were compared using t-test statistical analysis, with $p<0.05$.

\section{Statistical analysis}

Descriptive statistical measures were applied. We calculated percent of neurophysiological test that were affected in ALS patients, percent of abnormalities of anatomical MRI images, mean value of FA at different points of corticospinal tract and callossum corpus; mean value of fibre count of corticospinal tract and callosum corpus in ALS and health subjects. A regression analysis was done between FA and fibre count values and ALSFRS-R scale in ALS patients.

\section{Results}

\section{Clinical characteristics of the study population}

$25 \%$ of ALS patients had antecedents of exposure to toxic substances like: plumb, heavy metals, organophosphate substances in previous step of theirs life.

ALSFRS-R scale had a mean score of $38 / 48$ maximum value.
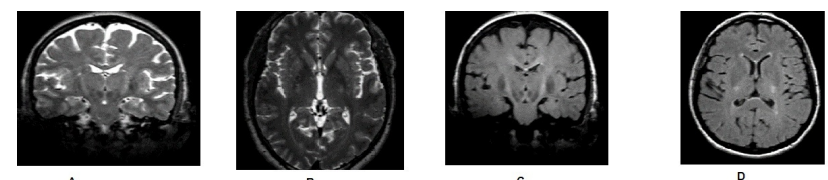

Figure 1 Abnormalities of anatomical MRI (3T). Hyperintendity of corticospinal tract in a patient with ALS at T2 sequences $(A, B)$ and FLAIR $(C, D)$, coronal view $(A, C)$ and axial view $(B, D)$.

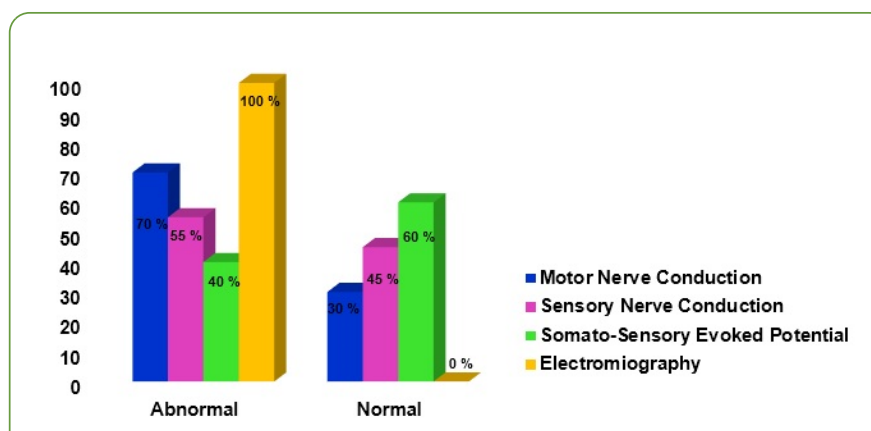

Graph 1 Percent of positivity of electrophysiological evaluation

\section{Neurophysiological studies}

MNC studies were: abnormal in $70 \%$ of ALS patients, principal abnormalities were amplitude diminish, latencies enlargement and nerve conduction velocities slowing. SNC studies were abnormal in $55 \%$ of ALS patients, principal abnormalities were: absence of responses, amplitude diminishes, latencies enlargement and nerve conduction velocities slowing. SSEP was abnormal in $40 \%$ of ALS patients; principal abnormalities were: P40 latency and Central Conduction Time enlargement. EMG were abnormal in $100 \%$ of ALS patients, it showed a neurogenic pattern in muscles of three anatomical regions (bulbar, cervical and lumbo-sacral) (Graph 1).

\section{Anatomical images}

Anatomical image showed hyper intensity of corticospinal tract in $50 \%$ of patients, at FLAIR and T2 sequences (Figure 1).

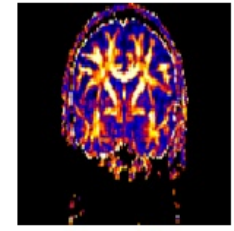

A

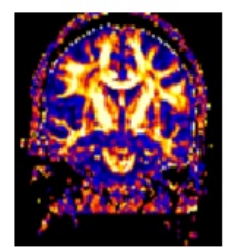

D

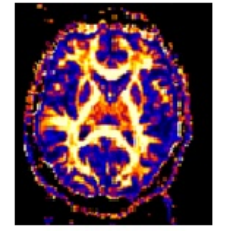

B

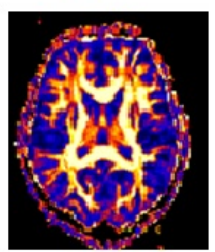

E

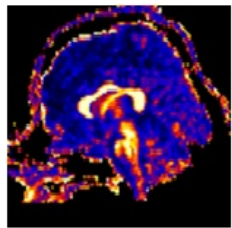

C

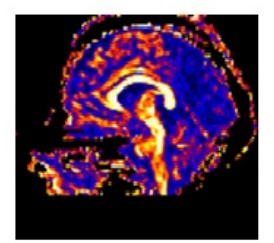

$\mathbf{F}$
Figure 2 Fractional anisotropy diminishes in a ALS patient $(A, B, C)$ in comparison with a health subject $(D, E, F)$. Coronal (A, D), Axial (B, E) and Sagital (C, F) views. 


\section{DTI images}

FA was diminished along entire corticospinal tract and corpus callosum in ALS patients in comparison with health subjects (Figure 2), but this diminish was not homogeneous, it was more meaningful at cortex $(p=0.00)$, internal capsule $(p=0.04)$ and corpus callosum (previous $p=0.04$ and posterior portions $p=0.00$ ) (Graph 2); $p<0.05$ was considered statistically significant.

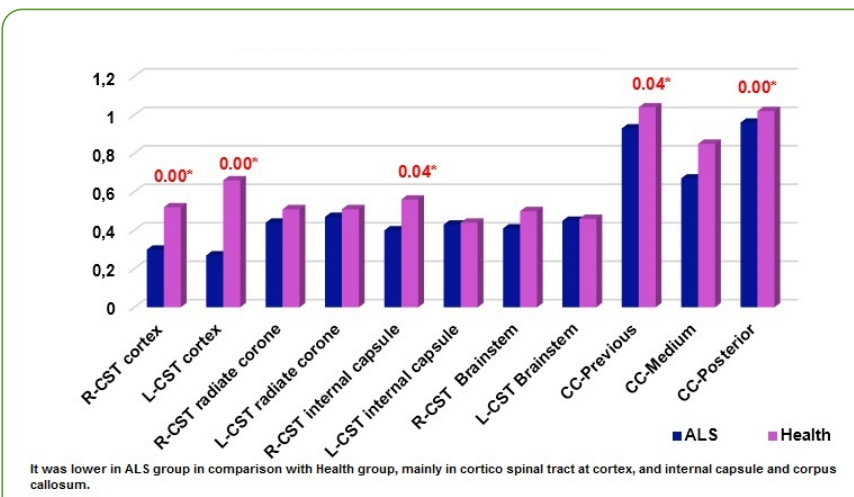

Graph 2 Fractional anisotropy means values.

\section{Tractography}

Tractography study revealed that CST volumes of ALS patients is lower than health subjects,

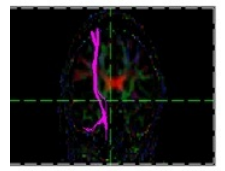

A
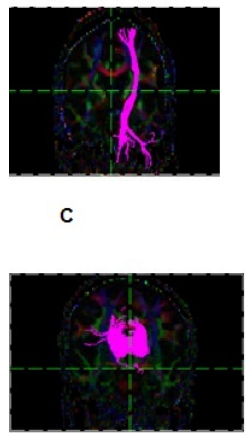
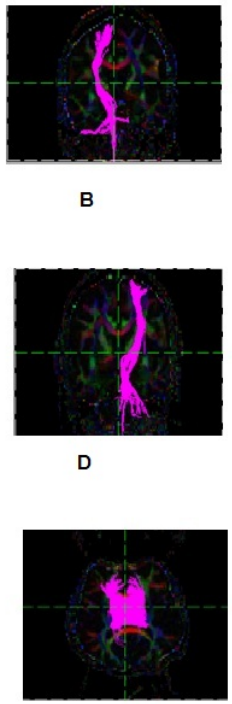

$\mathbf{F}$
Figure 3 Fiber count of right (A, B) and left (C, D) cortico spinal tract and corpus callosum (E, F). It was diminished in an $A L S$ patient $(A, C, E)$ in comparison with a health subject $(B, D, F)$.

means fibre count was lower at corticospinal tract and corpus callosum in ALS patients in comparison with health subjects, $p=0.01$ (Graph $\mathbf{3}$ and Figure 3); $p<0.05$ was considered statistically significant.

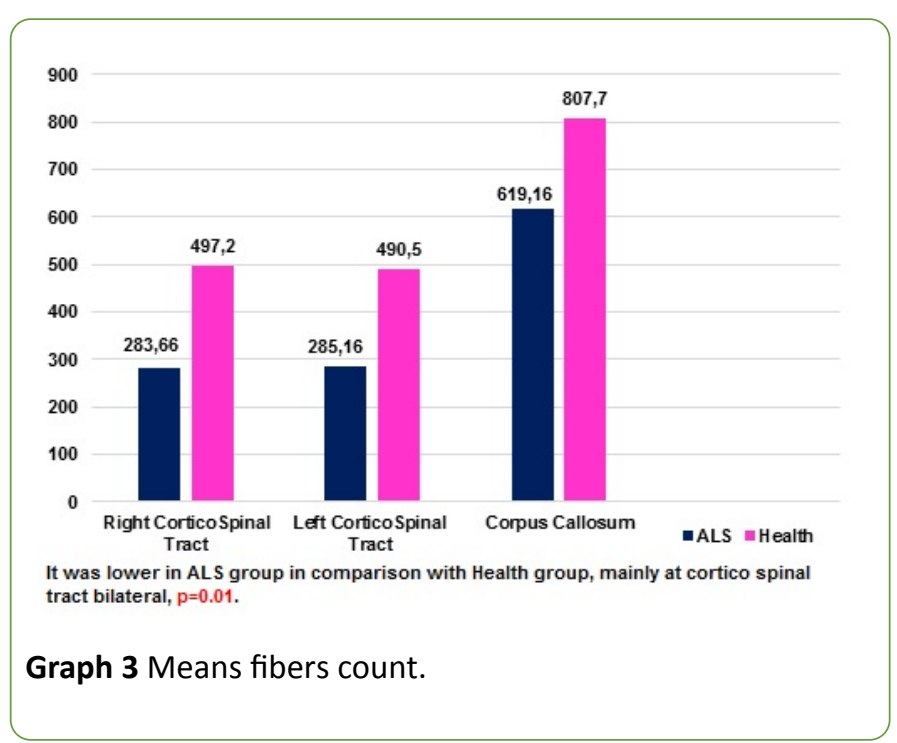

Regression analysis between FA at different points of corticospinal tract, fibre counts and ALSFRS-R scale was signified at right and left cortex $(p=0.00-0.0)$, right and left internal capsule $(p=0.02-0.00)$, left brainstem $(p=0.00)$, and corpus callosum (previous $p=0.00$, medium $p=0.02$ and posterior $p=0.00$ ) (Table 1 ); $p<0.05$ was considered statistically significant.

Table 1 Regression analysis between clinic (ALSFRS-R) scale and imagenological parameters.

\begin{tabular}{|l|l|l|l|}
\hline Parameters & Intercept (b) & $\begin{array}{l}\text { Std. } \\
\text { Error }\end{array}$ & P \\
\hline FA CST right cortex & 0.34 & 0.11 & 0.03 \\
\hline FA CST left cortex & 0.26 & 0.05 & 0.00 \\
\hline FA CST right radiated corone & 0.53 & 0.20 & 0.05 \\
\hline FA CST left radiated corone & 0.63 & 0.28 & 0.09 \\
\hline FA CST right internal capsule & 0.54 & 0.14 & 0.02 \\
\hline FA CST left internal capsule & 0.57 & 0.06 & 0.00 \\
\hline FA CST right brainstem & 0.48 & 0.19 & 0.06 \\
\hline FA CST left brainstem & 0.47 & 0.07 & 0.00 \\
\hline Corpus callosum previous & 1.11 & 0.05 & 0.00 \\
\hline Corpus callosum medium & 0.90 & 0.24 & 0.02 \\
\hline Corpus callosum posterior & 0.92 & 0.41 & 0.00 \\
\hline CST-right fiber count & 147.79 & 5161.50 & 0.41 \\
\hline CST-left fiber count & 519.54 & 0.40 \\
\hline Corpus callosum fiber count & & 0.40 \\
\hline
\end{tabular}

\section{Voxel based morphometry (VBM)}

Patterns of brain atrophy in grey matter: We observed reduced grey matter density in ALS patients in relation with control group in cingulate gyrus (anterior and medium 
portion), anterior portion of occipital lobe, left caudate and putamen nucleus, right claustrum nucleus, lower and medium temporal gyrus bilateral, left pre-central and post-central gyrus (Figure 4); $p<0.05$ was considered statistically significant.
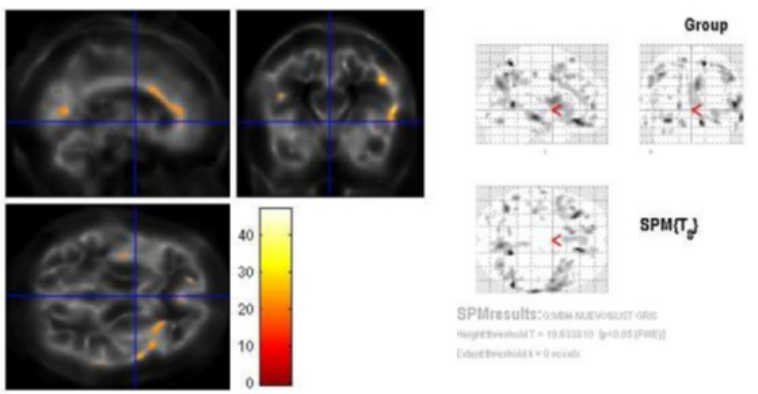

Figure 4 Generic MRI brain slices with superimposed areas showing statistically significant regions of grey matter atropy ( $p<0.05$, corrected for multiple comparisons) in a group of 20 ALS patients compared with 20 healthy agematched controls using voxel-based morphometry. It shows grey matter atropy in cingulate gyrus (anterior and medium portion), anterior portion of occipital lobe, left caudate and putamen nucleus, right claustrum lower and medium temporal gyrus bilateral, left precentral and post central gyrus. The colored bar represents the T score.
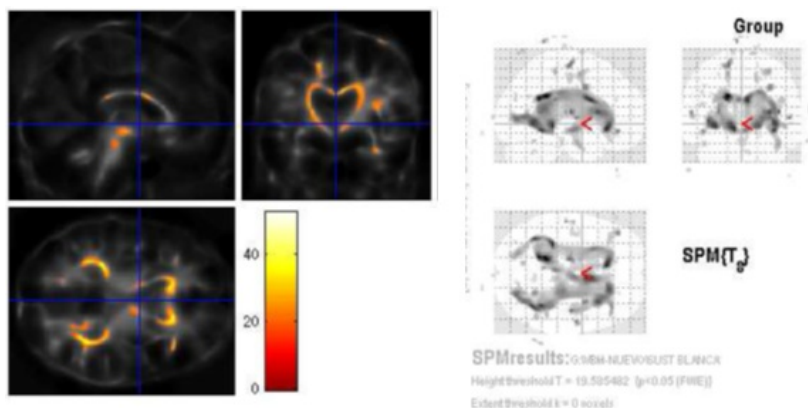

Figure 5 Generic MRI brain slices with superimposed areas showing statistically significant regions of white matter atropy ( $p<0.05$, corrected for multiple comparisions) in a group of 20 ALS patients compared with 20 healthy agematched controls using voxel-based morphometry. It shows grey matter atropy in corpus callosum (medium and posterior portion), corticospinal tract (at midbrain and pons), bilateral internal capsule (medium and posterior third), bilateral optical radiation, bilateral loer longitudial fascicle, bilateral hippocampal fimbriae, and bilateral radiated corona and pontocerebellar fibers. The colored bar represents the T score.

Patterns of brain atrophy in white matter: We observed reduced white matter density in ALS patients in relation with control group in corpus callosum (medium and posterior portion), corticospinal tract (at midbrain and pons), bilateral internal capsule (medium and posterior third), bilateral optical radiation, bilateral lower longitudinal fascicle, bilateral hippocampal fimbriae, and bilateral radiated corona and ponto-cerebellar fibres (Figure 5); $\mathrm{p}<0.05$ was considered statistically significant

\section{Discussion}

In ALS physiopathology some researchers have been postulated a disfunction of Superoxido Dismutasa 1 (SOD1) enzyme, it produce an increase of free radicals into the cell, wich produce abnormalities in the cell transport, dysfunction of the intacelular organelos increase of the glutamate levels into the cell, excitotoxicity, wich is mediated by glutamate and cell death. This proces ocurrs into the neurons and glias [3].

In ALS causes enviroment factors have been studied, such as: toxic exposure (heavy metals, calcium, selenium, manganesum, magnesium, mercury, lead, copper), cigar, organic solvents, organophosphorus substances, radiations, electromagnetic fields $[11,12]$.

There is limited evidence that those factors could cause ALS. In our cases serie only $25 \%$ reported contact with some toxic substance.

\section{Neurophysiological studies}

Motor nerve conduction studies show expected results, they were afected in $70 \%$ of ALS patients. In a great of group of ALS patients motor response of peripheral nerves are affected due to retrograde degeneration.

Sensory nerve conduction showed abnormalities in 55\% of ALS patients. The revised El Escorial (World Federation of Neurology) criteria for the diagnosis of ALS allow abnormal sensory NCS only in the presence of entrapment syndrome or coexisting peripheral nerve disease; normal electrophysiological studies on sensory nerves are generally required for the diagnosis of ALS. Nevertheless, several neurological, clinical neurophysiological and neuropathological studies have suggested that ALS is a more generalised neurodegenerative disorder [13].

Pugdahl reported of 41 sensory nerves examined in ALS, 32 (78\%) nerves had abnormal nerve conduction parameters: 6 had decreased of nerve conduction velocity and decreased of amplitude, 10 had decreased of nerve conduction velocity, 14 had decreased of amplitude and 2 nerves did not elicit any response [13].

Animals model of ALS have reported degeneration of proprioceptive dorsal root ganglion cell and nerve pathology in ventral and dorsal roots at the latest period of the disease and small nerve fiber pathology by inducing axonal stress in smalldiameter, swollen axons in the dorsal roots $[14,15]$.

SSEP were afeted in $40 \%$ of ALS patients. At past time this abnormality was an exclusion criteria for ALS, although there are some report from eighty decade that puts in evidence that the sensory system could therefore be affected by the same abnormality involving the motor neurons, but to a lesser extent and without clinical evidence. Cosi et al. in 1984 studied 47 ALS patiets, they reported N13 latency of SSEP significantly 
increased, central conduction time was significantly increased, mean value of N19 latency was significantly increased, P40 latencies were significantly increased, amplitude was significantly reduced in ALS patients in comparisson with control group [16].

At the actual times autopsy cases and animal studies showed involvement of sensory pathways, some researchs have demonstrate sensory abnormalities of the SSEP and spinal cord in ALS patients. Cohen, et al. have demostrated through multi-parametric MRI of the spinal cord DTI and magnetization transfer abnormalities in the lateral corticospinal tract and dorsal segments of the cervical spinal cord, spinal cord atrophy associated with the corresponding muscle deficit and correlations between FA and electrophysiological measurements $[17,18]$.

EMG was abnormal in all of our patients. It is used as a diagnostic criteria of ALS. EMG is very important to evidence of generalized damaged of moto neuron of anterior horn, it shows acute or chronic denervation, reinervation signs. Awaji criteria recommend giving importance to electrophysiologic evidence that shows chronic neurogenic changes at the same time that clinic evidence of motoneuron damage [19].

EMG let confirm ALS diagnosis if abnormalities raise to two or three anathomic regions and let do differential diagnosis [19].

\section{Anatomical images}

Da Rocha et al. found hyperintensity of corticospinal tract in ALS patients and not in health subjects. Authors attributed this finding to the loss of myelin and axons, secondary to neuronal degeneration $[20,21]$.

Others authors have reported hyperintensity of corticospinal tract, however, the sensitivity of such changes has been estimated at $<40 \%$ and the specificity $<70$ [22-29].

\section{DTI images}

In our study FA was diminished along entire corticospinal tract and corpus callosum in ALS patients in comparison with health subjects, but this diminish was not homogeneous, it was more meaningful at cortex, internal capsule and corpus callosum.

The value of FA was diminished in corticospinal tract in comparison with the normal value obtained in a big previous study of health subjects in Cuba [30].

The majority of DTI studies to date have used a region of interest method to compare groups, all published studies in corticospinal tract have demonstrated the potential of DTI to discriminate ALS patients from healthy controls on the basis of changes in FA at various levels of the corticospinal tract. FA changes within the CST at baseline may be even able to predict disability at 6 months according to some studies [31-33].

Loss of pyramidal motor neurons in the primary motor cortex and axonal degeneration of the corticospinal tract, together with the proliferation of glial cells, extracellular matrix expansion, and interneuron abnormalities, may contribute to the observed corticospinal tract DTI changes. The most pronounced decreased FA and increased have been shown in the posterior limb of internal capsule [34,35].

Verstraete et al. in 2010 reported that FA values in the corpus callosum and the corticospinal tract were found to be significantly reduced $(p<0.05)$ in ALS patients compared to controls. The FA reduction was more dispersed in the

Corpus callosum compared to the corticospinal tracts. Tractbased results, analysing the trajectory of FA along the corticospinal tracts, showed that the reduction in FA becomes less pronounced as the tract descends from the cortex to the brainstem corresponding to more loss of integrity in the rostral part compared to the caudal part of the corticospinal tract. It is accord with our results [36].

\section{VBM}

In some VBM study of ALS patient's widespread regions of grey matter atrophy were detected in both motor (primary motor cortex) and non-motor regions (including the temporal lobes). [27-30,36].

Other studies have demonstrated atrophy in the bilateral para-central lobule and in non-motor structures like: the corpus callosum, the cerebellum, and the frontotemporal and occipital areas [37-39].

Canu et al. on 2011 reported significant volume losses in white matter areas: left superior frontal region, the vicinities of the supplementary motor area bilaterally, right precentral region, and left inferior temporal region [27].

We don't find any abnormality of FA or VBM at cerebellum in ALS patients, nevertheless several researches have reported diminish of FA in cerebellum and atrophy of grey and white matter in specific zones of cerebellum in ALS patients [40].

Grolez et al. on 2016 published an important review in relation with the value of $\mathrm{MRI}$ as a biomarker for amyotrophic lateral sclerosis. They have mentioned grey matter atrophy in ALS patients in some areas like: pre-central gyrus, frontal lobe, the cingulate cortex, several parts of the temporal cortex, the hippocampus, the parietal cortex (mostly in the post-central cortex) and the insula, they have mentioned too occipital grey matter and cerebellar atrophy, but, less common. In relation with basal ganglia they refered thalamus and the caudate nucleus atrophy [39].

In a meta-analysis made by Shen et al., who analized 29 studies, was evidenced that grey matter was decreased in ALS patients compared with health controls, it was more significant in the bilateral Rolandic operculum, right precentral gyrus, left lenticular nucleus (mainly the putamen) and right anterior cingulate gyri, with a thresholdof $p<0.005$. The metaregression analyses showed that higher symptom severity (proportional ALSFRS scores) was associated with decreased grey matter in the right precentral gyrus and the left inferior frontal gyrus. A longer disease duration correlated with more grey matter atrophy in the right precentral gyrus in patients with ALS [40]. 
Unfortunately we don't evaluate functional conectivity. In relation with it, Verstraete et al. on 2011 demosntrated a single impaired sub-network in ALS patients, it was consisted of 9 nodes and 10 (bidirectional) connections, of reduced connectivity in patients with ALS (NBSa; $p=0.0108$, permutation testing). This impaired network overlapped with the left and right precentral gyrus, left pallidum, left hippocampus, left and right caudal middle frontal gyrus, right paracentral gyrus, right posterior cingulate and right precuneus. Interestingly, although a whole brain analysis was performed without any a priori selection of motor regions, the regions of this impaired network strongly overlap with regions that are known to play a key role in motor movement and control [41].

\section{Correlation between FA and ALSFRS-R score}

The opinion of the authors are very controversial in relation with this point.

Trojsi and Verstraete et al. in 2010 did not find a significant correlation using Pearson's correlation testing between imaging measures (averaged along the cortico spinal tract) and clinical markers (ALSFRS-R) [34-36].

Verstraete et al. in 2011 showed that diminish of conectivity in ALS patients were not significantly correlated ALSFRS or progression rate scores [42].

Grolez et al. in 2016 mentioned that lower ALSFRS-R scores were associated with greater volume loss in the basal ganglia and volume changes in several parts of the frontal lobe; and the disease duration was negatively correlated with FA in the cerebellum, the subcortical white matter of insula, the ventrolateral premotor cortex, the cingulum, the precuneus and the splenium of the corpus callosum [39].

As Kim et al. research in 2017, ALSFRS-R score was associated with atrophy in multifocal gray matter areas including the bilateral frontal, left superior, supramarginal gyri and the left orbitofrontal area [41].

\section{Conclusion}

Motor and Sensory neurophysiological abnormalities could be found in ALS patients. MRI image is very useful in evaluate brain structures of ALS patients. This study found that sporadic ALS patients with normal levels of cognition and pure motor symptoms show multiple sites of cortical and subcortical atrophy in areas that extend beyond motor regions.

\section{Declaration of Interest}

The authors report no conflicts of interest.

\section{References}

1. Kiernan MC, Vucic S, Cheah BC, Turner MR, Eisen A, et al. (2011) Amyotrophic lateral sclerosis. Lancet 377: 942-955.

2. Hernández A (2016) ALS diagnostic in the electrodiagnostic department of an orthopedic hospital during 2014-2015. Clinica and Electrophysiological Characteristics. Open Access J Neurol Neurosurg 1: 555-557.

3. Ruiz RAL, Clavijo GD, Ramón MO, Ruiz M, García CA, et al. (2006) Bases biológicas y patobiológicas humanas de la esclerosis lateral amiotrófica. Universitas Médica 47: 35-54.

4. Menke RAL, Gray E, Lu Ch H, Kuhle J, Talbot K, et al. (2015) CSF neurofilament light chain reflects corticospinal tract degeneration in ALS. Ann Clin Transl Neurol 2: 748-755.

5. Feneberg E, Gray E, Fischer S, Gordon D, Thezenas ML (2017) TDP-43 based biomarker development in ALS. Amyotroph Lateral Scler Frontotemporal Degener 18: 187-199.

6. Adiutori R, Aarum J, Zubiri I, Leoni E, Di Benedetto P, et al. (2017) Circulating neurofilament-containing hetero-aggregates as a test-bed for novel biomarkers and therapeutics in neurodegeneration. Amyotroph Lateral Scler Frontotemporal Degener 18: 187-199.

7. Thompson A, Gray E, Mager I, Fischer R, Thezenas ML, et al. (2017) Characterisation of CSF extracellular vesicles and their proteome in ALS. Amyotroph Lateral Scler Frontotemporal Degener 18: 187-199.

8. Basso M, Passeto L, D' Agostino V, Maiolo D, Baldelli Bombelli F, et al. (2017) Circulating exosomes as a novel source of biomarkers for ALS progression. Amyotroph Lateral Scler Frontotemporal Degener 18: 187-199.

9. Aricha R, Cudkowicz M, Berry J, Windebank A, Staff N, et al. (2017) Chitotriosidase as a biomarker for ALS. Amyotroph Lateral Scler Frontotemporal Degener 18:187-199.

10. Brenner D, Hiergeist A, Adis C, Gessner A, Ludoph A, et al. ( 2017) The fecal microbiome ALS patients. Amyotroph Lateral Scler Frontotemporal Degener 18: 187-199.

11. González-Mingot $C$, Sánchez-Monge IR, Purroy F, Solana-Moga MJ, Peralta-Moncusí S, et al. (2017)Influencia de los factores ambientales-analíticos sobre el fenotipo de esclerosis lateral amiotrófica en un medio rural. Rev Neurol 65: 203-208.

12. Huss A, Spoerri A, Egger M, Kromhout H, Vermeulen R (2015) The Swiss National Cohort: Occupational exposure to magnetic fields and electric shocks and risk of ALS: The Swiss National Cohort. Amyotroph Lateral Scler Frontotemporal Degener 16: 80-85.

13. Pugdahl K, Fuglsang-Frederiksen A, De Carvalho M, Johnsen B, Fawcett PRW, et al. (2007) Generalised sensory system abnormalities in amyotrophic lateral sclerosis: A European multicentre study. J Neurol Neurosurg Psychiatry 78: 746-749.

14. Sábado J, Casanovas A, Tarabal O, Hereu M, Piedrafita L, et al. (2014) Accumulation of misfolded SOD1 in dorsal root ganglion degenerating proprioceptive sensory neurons of transgenic mice with amyotrophic lateral sclerosis. BioMed Research International.

15. Sassone J, Taiana M, Lombardi R, Porretta-Serapiglia C, Freschi $M$, et al. (2016) ALS mouse model SOD1G93A displays early pathology of sensory small fibers associated to accumulation of a neurotoxic splice variant of peripherin. Human Molecular Genetics 25: 1588-1599.

16. Cosi V, Marco P, Mazzini L, Callieco R (1984) Somatosensory evoked potentials in amyotrophic lateral sclerosis. Journal of Neurology, Neurosurgery and Psychiatry 47: 857-861.

17. Cohen-Adad J, Mounir-El-Mendili M, Morizot-Koutlidis R, Lehéricy S, Meininger V (2013) Involvement of spinal sensory pathway in ALS and specifi city of cord atrophy to lower motor 
neuron degeneration. Amyotroph Lateral Scler Frontotemporal Degener 14: 30-38.

18. Fang $X$, Zhang $Y$, Wang $Y$, Zhang $Y$, Hu J, et al. (2016) Disrupted effective connectivity of the sensorimotor network in amyotrophic lateral sclerosis. J. Neurol 263: 508-16.

19. De Carvalho M, Awaji SM (2009) Diagnostic algorithm increases sensitivity of El-Escorial criteria for ALS diagnosis. Amyotroph Lateral Scler 10: 53-57.

20. Da Rocha AJ, Oliveira AS, Fonseca RB, Maia Jr AC, Buainain RP, et al. (2004) Detection of corticospinal tract compromise in amyotrophic lateral sclerosis with brain MR imaging: relevance of the T1-weighted spin-echo magnetization transfer contrast sequence. AJNR Am J Neuroradiol 25: 1509-1515.

21. Álvarez-Uría Tejero MJ, Sáiz Ayala A, Fernández Rey $C$ Santamarta Liébana ME, Costilla García S (2011) Diagnóstico de la esclerosis lateral amiotrófica: avances en RM. Radiología. 53: 146-155.

22. Turner MR, Modo M (2010) Advances in the application of MRI to amyotrophic lateral sclerosis. Expert Opin Med Diagn 4: 483-496.

23. Kwan JY, Jeong SY, Van Gelderen P, Deng HX, Quezado MM, et al. (2012) Iron accumulation in deep cortical layers accounts for MRI signal. Abnormalities in ALS: Correlating 7 Tesla MRI and Pathology. PLoS ONE 7: 35241.

24. Müller HP, Agosta F, Riva N, Spinelli EG, Comic G, et al. (2018) Fast progressive lower motor neuron disease is an ALS variant: $A$ two-centre tract of interest-based MRI data analysis. Neurolmage Clinical 17: 145-152.

25. Agosta F, Chio 'A, Cosottini M, De Stefano N, Falini A (2010) The present and the future of neuroimaging in amyotrophic lateral sclerosis. AJNR Am J Neuroradiol 31: 1769 -1777.

26. Turner MR and Verstraete E (2015) What does imaging reveal about the pathology of amyotrophic lateral sclerosis? Curr Neurol Neurosci Rep 15: 45.

27. Canu E, Agosta F, Riva N, Sala S, Prelle A, et al. (2011) The topography of brain microstructural damage in amyotrophic lateral sclerosis assessed using diffusion tensor MR imaging. AJNR Am J Neuroradiol 32: 1307-1314.

28. Huynh W, Simon NG, Grosskreutz J, Turner MR, Vucic S, et al. (2016) Assessment of the upper motor neuron in amyotrophic lateral sclerosis. Clinical Neurophysiology 127: 2643-2660.

29. Kollewe K, Körner S, Dengler R, Petri S, Mohammadi B (2012) Magnetic resonance imaging in amyotrophic lateral sclerosis. Neurology Research International.

30. Góngora D, Domínguez M and Bobes MA (2016) Characterization of ten white matter tracts in a representative sample of Cuban population. BMC Medical Imaging 16: 59-70.
31. Simon NG, Turner MR, Vucic S, Al-Chalabi A, Shefner J, et al. (2014) Quantifying disease progression in amyotrophic lateral sclerosis. Ann Neurol 76: 643-657.

32. Berger $H$ (2013) The involvement of the cerebellum in amyotrophic lateral sclerosis. Amyotroph Lateral Scler Frontotemporal Degener 14: 507-515.

33. Müller HP, Unrath A, Huppertz HJ, Ludolph AC, Kassubek J et al. (2012) Neuroanatomical patterns of cerebral white matter involvement in different motor neuron diseases as studied by diffusion tensor imaging analysis. Amyotrophic Lateral Sclerosis 13: 254-264.

34. Trojsi F, Corbo D, caiazzo G, Piccirillo G, Monsurr MR, et al. (2013) Extramotor neurodegeneration in amyotrophic lateral sclerosis: A 3T high angular resolution diffusion imaging. Amyotroph Lateral Scler Frontotemporal Degener 14: 553-561.

35. Verstraete E, Polders DL, Mandl RCW, Van Den Heuvel MP, Veldink JH, et al. (2014) Multimodal tract-based analysis in ALS patients at 7T: A specific white matter profile? Amyotroph Lateral Scler Frontotemporal Degener 15: 84-92.

36. Verstraete E, Van den Heuvel MP, Veldink JH, Blanken N, Mandl RC, et al. (2010) Motor network degeneration in amyotrophic lateral sclerosis: A structural and functional connectivity study. PLOS ONE 5: 13664.

37. Douaud GI, Filippini N, Knight S, Talbot K, Turner MR (2011) Integration of structural and functional magnetic resonance imaging in amyotrophic lateral sclerosis. Brain 134: 3470-3479.

38. Menke RAL, Proudfoot M, Wuu J, Andersen PM, Talbot K, et al. (2016) J Neurol Neurosurg Psychiatry 87: 580-588.

39. Grolez G, Moreau C, Danel-Brunaud V, Delmaire C, Lopes R, et al. (2016) The value of magnetic resonance imaging as a biomarker for amyotrophic lateral sclerosis: a systematic review. Neurology 16: 155-172.

40. Shen D, Cui L, Fang J, Cu iB, Li V (2016) Voxel-wise meta-analysis of grey matter changes in amyotrophic lateral sclerosis. Front. Aging Neurosci 8: 64.

41. Kim HJ, De Leon M, Wang X, Kim HY, Lee YJ, et al. (2017) Relationship between clinical parameters and brain structure in sporadic amyotrophic lateral sclerosis patients according to onset type: A voxel-based morphometric study. PLoS One 12: 0168424.

42. Verstraete E, Veldink JH, Mandl RCW, Van den Berg LH, Van den Heuvel MP (2011) Impaired structural motor connectome in amyotrophic lateral sclerosis. PLoS One 6: 24239. 